\title{
Gradient Method for Finding Optimal Scheduling in Infinite Dimensional Models of Chemotherapy
}

\author{
JAROSLAW SMIEJA*, ANDRZEJ SWIERNIAK and ZDZISLAW DUDA \\ Institute of Automation. Silesian Technical University Akademicka 16, 44-100 Gliwice, Poland
}

(Received November, 1998; In final form March 20, 2000)

\begin{abstract}
One of the major obstacles against succesful chemotherapy of cancer is the emergence of resistance of cancer cells to cytotoxic agents. Applying optimal control theory to mathematical models of cell cycle dynamics can be a very efficient method to understand and, eventually, overcome this problem. Results that have been hitherto obtained have already helped to explain some observed phenomena, concerning dynamical properties of cancer populations. Because of recent progress in understanding the way in which chemotherapy affects cancer cells, new insights and more precise mathematical formulation of control problem, in the meaning of finding optimal chemotherapy, became possible. This, together with a progress in mathematical tools, has renewed hopes for improving chemotherapy protocols. In this paper we consider a population of neoplastic cells stratified into subpopulations of cells of different types. Due to the mutational event a sensitive cell can acquire a copy of the gene that makes it resistant to the agent. Likewise, the division of resistant cells can result in the change of the number of gene copies. We convert the model in the form of an infinite dimensional system of ordinary differential state equations discussed in our previous publications (see e.g. Swierniak et al., 1996b; Polański et al. 1997; Swierniak et al., 1998c), into the integro-differential form. It enables application of the necessary conditions of optimality given by the appropriate version of Pontryagin's maximum principle, e.g. (Gabasov and Kirilowa, 1971). The performance index which should be minimized combines the negative cumulated cytotoxic effect of the drug and the terminal population of both sensitive and resistant neoplastic cells. The linear form of the cost function and the bilinear form of the state equation result in a bang-bang optimal control law. To find the switching times we propose to use a special gradient algorithm developed similarly to the one applied in our previous papers to finite dimensional problems (Duda 1994; 1997).
\end{abstract}

Keywords: gene amplification, cancer chemotherapy, infinite dimensional systems

\section{INTRODUCTION}

Despite a long history of mathematical modeling of cancer chemotherapy its practical application to development of chemotherapy protocols has been arguably negligible (with minor exceptions). However, one cannot underestimate its importance in the development of ideas of chemotherapy scheduling, multidrug protocols, and recruitment.

*e-mail: jsmieja@ia.polsl.gliwice.pl 
In this paper, we would like to move the practical importance of mathematical modeling a step forward. Our previous experience, concerned asymptotic analysis of particular models of cancer populations that lead to better understanding of previously unexplained phenomena (Świerniak et al. 1996a; 1998b; 1998c, Polański et al. 1997), On the basis an approach to developing optimal chemotherapy scheduling is presented and its meaning for clinical procedures is discussed.

Recent experimental results show that although the entire DNA content should remain unchanged at each mitotic cell division, some fraction of it actually undergoes continuous change as far as its amount per cell and structure are concerned. That change can take the form of a process referred to as gene amplification, which includes an increase in the number of copies of a gene which codes for a protein that aides either removal or metabolization of the drug. The more copies of the gene present, the more resistant the cell, in the sense that it can survive under higher concentrations of the drug.

Gene amplification can be enhanced by conditions that influence DNA synthesis. Increased number of gene copies can produce even more gene products, which, if they confer resistance to chemotherapeutic drugs, leads to evolving resistance in the cancer population. Increase of drug resistance by gene amplification has been observed in numerous experiments with in vivo and cultured cell populations. For further references, see publications (Stark, 1993) and (Windle and Wahl, 1992).

The emergence of resistance to chemotherapy has been first considered in a point mutation model of Coldman and Goldie (1983) and then in the framework of gene amplification by Agur and Harnevo (1991; 1992; 1993). The main idea is that there exist spontaneous or induced mutations of cancer cells towards drug resistance and that the scheduling of treatment should anticipate these mutations. The point mutation model can be translated into simple recommendations, which have even been recently tested in clinical trials. The gene amplification model was extensively simulated and also resulted in recommendations for optimized therapy.
Numerous experiments proved that the process of gene amplification may be reversible (i.e. cells with increased number of gene copies tend to become extinct) whereas, in some cases, it is stable (i.e. the amplification persisted even after the selective agent causing it has been removed) (Kaufman et al, 1981). We present a model of chemotherapy based on a stochastic approach to evolution of cancer cells. Asymptotic analysis of this model, which has been proposed and broadly discussed in our previous works, helps to explain that phenomena and other dynamical properties (Świerniak et al. 1996a; 1998c, Polański et al. 1997).

In addition it has been established that, at least in some experimental systems, tumor cells may increase the number of copies of an oncogene in response to an unfavorable environment. For further details, see a discussion by Kimmel and Axelrod (1990).

Mathematical modeling of gene amplification has provided good fits to experimental data (Axelrod et al. 1993, Harnevo and Agur 1991; 1992; 1993, Kimmel and Axelrod 1990, Kimmel et al. 1992, Kimmel and Stivers 1994). These results suggest that drug resistance and other processes altering the behavior of cancer cells may be better described by multistage mechanisms, including a gradual increase in number of discrete units, rather than by classical irreversible mutation models described by Coldman and Goldie (1979; 1983). For example, models with gene amplification predict the observed pattern of gradual loss of resistance in cancer cells placed in a non-toxic medium, see references (Brown et al., 1981) and (Kaufman et al., 1981).

The multistage stepwise model of gene amplification or, more generally, of transformations of cancer cells, leads to new mathematical problems and results in novel dynamic properties of the systems involved. These problems were first studied mathematically in (Kimmel and Axelrod, 1990) for the discrete-time models and in (Kimmel and Stivers, 1994) for the continuous-time models.

The model is broadly discussed in the next section and some main results are recalled. The description is completed with new results, alowing to analyze the system with any finite initial conditions. Afterwards, 

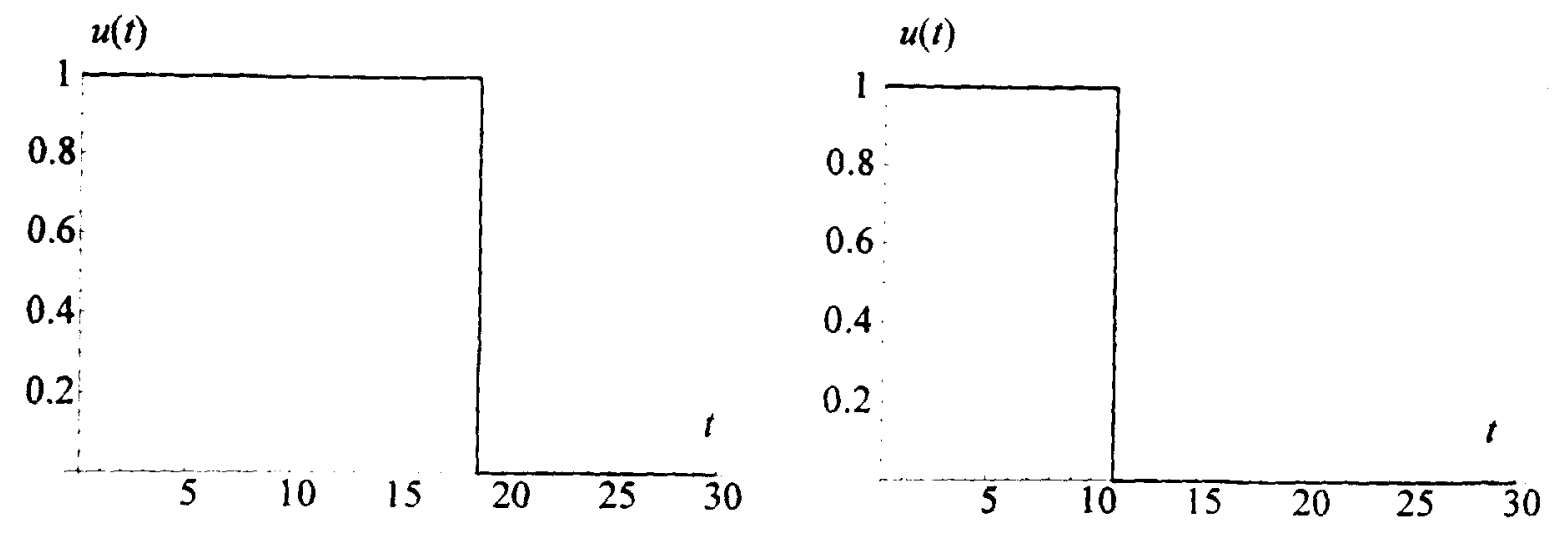

FIGURE 1 The optimal control for $M=1, r=0.1$ (left) and $\mathrm{r}=0.5$ (right)

the main idea of transforming the model into an integro-differential one is presented. In section 4 the optimization problem is stated. The particular forms of both the model and the performance index allow us to effectively apply the Pontryagin maximum principle and derive necessary contitions for the optimal control. Subsequently, a gradient method for finding the optimal solution is presented. Although gradient methods are one of the standard approaches to optimization problems, they are usually used for finding continuous control, whereas the solution to the problem stated in the paper requires control in the bang-bang form. Moreover, contrary to our previous works, the proposed method concerns infinite dimensional case and is modified to shorten computation time. In the final section, conclusions are drawn concerning the importance and applicability of the solution.

\section{THE INFINITE DIMENSIONAL MODEL}

Mathematical modeling of cancer populations taking into account both stochastic changes in number of gene copies in cells from one generation to another and the stochastic variability of cell lifetime can be based on branching random walk (Kimmel and Axelrod, 1990; Kimmel and Stivers, 1994). This approach leads to an infinite system of differential equations which may be used to model controling a cell population with evolving drug resistance caused by gene amplification or other mechanisms. It can be also understood as a mathematical variation of the model used by Harnevo and Agur (1992). Moreover, the model is general enough to accommodate different interpretations.

Let us consider a population of cells of types $i=0,1,2 \ldots$.Cells of type 0 are sensitive to the agent, whereas the types $i=1,2, \ldots$ consist of resistant cells of increasing level of resistance (for example, with increased number of DHFR or CAD gene copies per cell). It is also assumed that:

1. The lifespans of all cells are independent exponentially distributed random variables with means $1 / \lambda_{i}$ for cells of type $i$.

2. A cell of type $i \geq 1$ may mutate in a short time interval $(t, t+d t)$ into a type $i+1$ cell with probability $b_{i} d t+o(d t)$ and into type $i-1$ cell with probability $d_{i} d t+o(d t)$. A cell of type $i=0$ may mutate in a short time interval $(t, t+d t)$ into a type 1 cell with probability $\alpha d t+o(d t)$, where $\alpha$ is several orders of magnitude smaller than any of $b_{i} s$ or $d_{i} s$.

3. The chemotherapeutic agent affects cells of different types differently. It is assumed that its action 
results in a fraction $u_{i}$ of ineffective divisions (leading to abortion) in cells of type $i$.

4. The process is initiated at time $t=0$ by a population of cells of different types.

The postulated relationship for the rate $\alpha$ of the primary amplification event can be written as follows

$$
\alpha<<\min \left(d_{i}, b_{i}\right), \quad i \geq 1 .
$$

Generally, in view of the subcriticality of the process it seems reasonable to assume

$$
d_{i}>b_{i}, \quad i \geq 1 \text {. }
$$

Also, the following relationships between $b_{i} s$ and $d_{i} s$ seem to be justified by the intuition that cells overloaded with amplified gene copies may acquire new copies with more difficulty and lose them easier:

$$
d_{i+1} \geq d_{i}, \quad b_{i+1} \leq b_{i}, \quad i \geq 1 .
$$

As postulated by Schimke (see e.g. Kaufman et al, 1981; Brown et al., 1981), cells with more copies of the drug resistance gene may proliferate slower, i.e..

$$
\lambda_{i+1} \leq \lambda_{i}, \quad i \geq 0 .
$$

Since the drug influence on resistant cells is significantly smaller than on sensitive ones, we might assume all $u_{i}$ s equal zero except for $u_{0}$ denoted $u$ from now on. Moreover, since the differences in amplification/deamplification probablilities for resistant cells and cell lifespans are not significant, it seems justifiable to make additional assumptions that parameters $\lambda_{i}, \mathrm{~b}_{i}$ and $\mathrm{d}_{i}$ does not differ for cells of different type.

Let $N_{i}(t)$ denote the expected number of cells of type $i$ at time $t$. This leads to the following infinite system of differential equations:

$$
\left\{\begin{array}{ccc}
\dot{N}_{0}(t)= & {[1-2 u(t)] \lambda N_{0}(t)} \\
& & -\alpha N_{0}(t)+d N_{1}(t), \\
\dot{N}_{1}(t)= & \lambda N_{1}(t)-(b+d) N_{1}(t) \\
& & +d N_{2}(t)+\alpha N_{0}(t), \\
\dot{N}_{i}(t)= & \lambda N_{i}(t)-(b+d) N_{i}(t) \\
& \ldots & +d N_{i+1}(t)+b N_{i-1}(t), \quad i \geq 2,
\end{array}\right.
$$

where

$$
0 \leq u(t) \leq 1
$$

Based on gene amplification studies, there exist three phases in the evolution of the resistance process:

- The relatively rare primary event, i.e. the establishment of the founder cell of the resistant clone containing at least one unstable copy of the target gene (the probability of this event, per cell division, corresponds to the ratio $\alpha / \lambda$ in (5)).

- Subsequent amplification and deamplification events, occurring at high rates compared to $\alpha / \lambda$, resulting from instability of the amplified gene (the probabilities of these events, per cell division, correspond to the ratios $b / \lambda$ and $d / \lambda$ in (5)).

- Possible stabilization of the resistant phenotype, by integration of the amplified gene in the chromosomal structures (no counterpart in (5)).

A number of researchers have carried out the procedure to estimate rates of emergence and evolution of resistance (by gene amplification and other means) (Morrow, 1970; Varshaver et al., 1983; Murnane and Yezzi, 1988; Tlsty et al., 1989), obtaining estimates of the mutation probabilities, per cell division, in the range from $10^{-8}$ to $10^{-6}$, with generally higher estimates for tumorogenic than for "normal" cells. The data from the above papers were re-analyzed in a recent paper by Kimmel and Axelrod (1994), using a two-stage model of mutation. Although the estimates of primary event probabilities remain mostly unchanged, the probabilities of second stage forward and backward mutation are much higher, comparable to the estimates of amplification and deamplification probabilities(approximately 0.02 and 0.10 , respectively) obtained in (Brown et al., 1981; Kaufman et al., 1981; Kimmel and Axelrod, 1990; Kimmel and Stivers, 1994).

It is possible to derive a formula for calculating the number of cells in the whole resistant population. Denoting $N_{\Sigma}(t)=\Sigma_{i \geq 1} N_{i}(t)$, the following result has been obtained using the methods of Kimmel and Stivers (1994) in the case when the sensitive subpopulation is completely destroyed by the anticancer drug. 

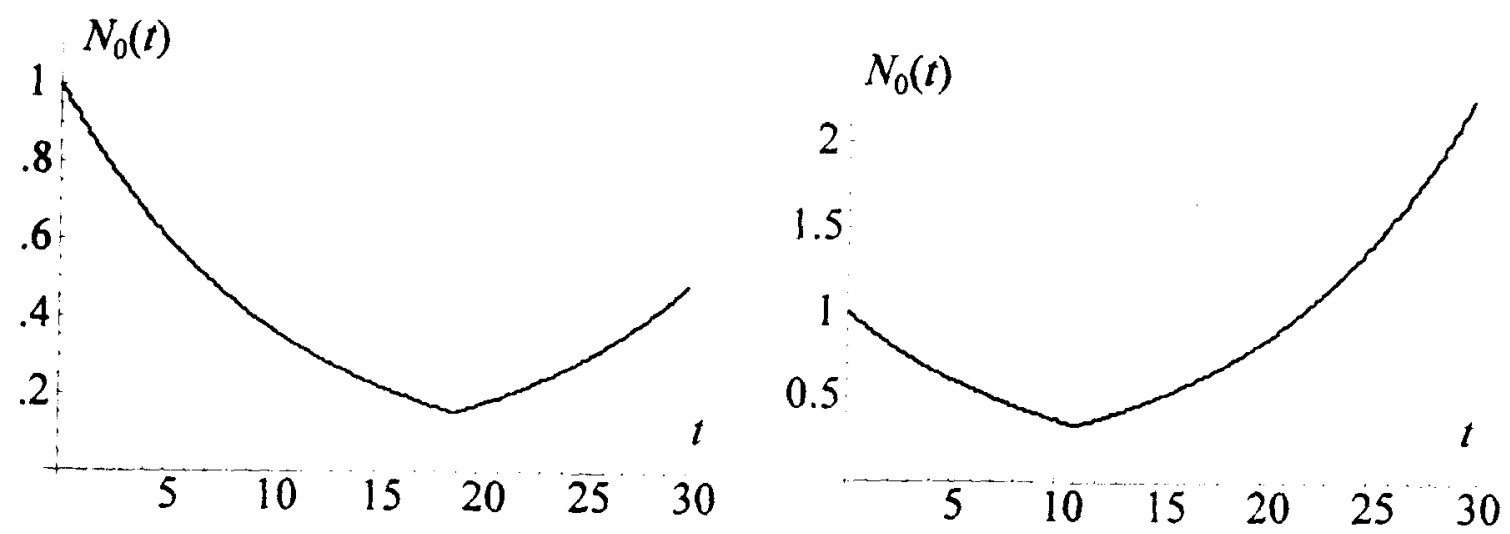

FIGURE 2 The dynamics of the sensitive subpopulation for $\mathrm{M}=1, \mathrm{r}=0.1$ (left) and $\mathrm{r}=0.5$ (right)

Suppose that $N_{i}(0)=\delta_{i 1}$ (meaning that $N_{1}(0)=1$ and $N_{i}(0)=0$ for $\left.i \neq 1\right)$ and $d \neq b$. Then

$$
\begin{aligned}
& N_{\Sigma}(t)=N_{\Sigma}^{1}(t)=\exp (\lambda t)- \\
& \exp (\lambda t) \sqrt{d / b} \int_{0}^{t} \frac{I_{1}(2 \sqrt{b} d \tau)}{\tau} \exp [-(b+d) \tau] d \tau
\end{aligned}
$$

where $I_{1}(t)$ is the modified Bessel function of order 1 (Abramowitz and Stegun, 1964) and $N_{\Sigma}^{1}(t)$ denotes number of cells in the subpopulation initiated by cells of the first type.

Using an asymptotic expansion of (7) it has been found (Polanski et al., 1997) that, assuming subcriticality of the process $(b<d)$, a condition for the decay of the resistant subpopulation is given by:

$$
\sqrt{\lambda}<\sqrt{b}-\sqrt{d} .
$$

Moreover we have (Polański et al., 1997):

$N_{1}(t)=N_{1}^{1}(t)=\sqrt{\frac{1}{d b}} I_{1}(2 \sqrt{b d} t) \exp [-(b+d-\lambda) t]$,

where $N_{1}^{L}(t)$ denotes number of cells of the first type in the subpopulation initiated by cells of the first type.

In (Polański et al., 1997) it was supposed that it was possible to analyse the system also in the case of other initial conditions. Indeed, following the same line of reasoning, in case of $N_{i}(0)=\delta_{i k}\left(N_{k}(0)=1\right.$ and
$N_{i}(0)=0$ for $\left.i \neq k\right)$ one can obtain the following formulae for $N_{\Sigma}(t)$ and $N_{1}(t)$ :

$$
\begin{aligned}
& N_{\Sigma}^{k}(t)=\exp (\lambda t)- \\
& k \exp (\lambda t)(\sqrt{d / b})^{k} \int_{0}^{t} \frac{I_{k}(2 \sqrt{b d} \tau)}{\tau} \exp [-(b+d) \tau] d \tau
\end{aligned}
$$

$$
N_{1}^{k}(t)=k \frac{1}{d}(\sqrt{d / b})^{k} \frac{I_{k}(2 \sqrt{b d} t)}{t} \exp [-(b+d-\lambda) t]
$$

where $I_{k}(t)$ is the modified Bessel function of $\mathrm{k}$-th order.

Under the assumption about total destruction of the sensitive subpopulation the model is linear, hence the equations (10) and (11) can be used to find $N_{\Sigma}(t)$ and $N_{1}(t)$ in the case of any finite non-zero initial conditions.

In the case when the assumption about total destruction of the sensitive subpopulation is not satisfied, the model can be considered as a system with positive feedback ('́wierniak et al., 1998a), whereas if the assumption is true, it may be treated as an open loop system. Using the Nyquist criterion for infinite dimensional systems and constant $u$ it has been found (Świemiak et al., 1998c) that in this case the condition of convergence of the whole population to zero is given by: 


$$
u>\frac{1}{2}+\frac{\alpha}{d} \cdot \frac{1}{d-b-\lambda+\sqrt{(b+d-\lambda)^{2}-4 b d}}
$$

Model (5) may be used to find the optimal control which minimizes an appropriate performance index, e.g..

$$
\begin{aligned}
J & =\sum_{i>0} N_{i}(T)+r \int_{0}^{T} u(t) d t \\
& =N_{0}(T)+N_{\Sigma}(t)+r \int_{0}^{T} u(t) d t
\end{aligned}
$$

In biological terms, the effect of the optimal control is minimization of the number of cancer cells at the end of the assumed therapy interval $[0, \mathrm{~T}]$ (all, as in (13), or only resistant ones - then the $N_{0}$ component should be omitted in the performance index), combined with minimization of the cumulative negative effects of the drug upon the normal tissues; $r$ is a weighing coefficient.

A straightforward approximation by finite truncation is improper because the important features of the infinite systems are ignored in this case, which has been discussed e.g. in (Polański et al., 1997). Our approach, however, consists in transforming the infinite dimensional description into one integro-differential equation without any loss of model properties. Afterwards, necessary conditions for optimal control could be found using the maximum principle.

\section{THE INTEGRO-DIFFERENTIAL MODEL}

The assumption about complete annihilation of the sensitive population is an overidealization. On the contrary, a constant influx from the sensitive compartment to the resistant one (and vice versa) should be expected. Let us assume only a finite number of nonzero initial condition elements in (5). Then system (5) can be transformed into a form of integro-differential equation. Particularly, in case of zero initial conditions of the whole resistant population $\left(N_{i}(0)=\delta_{i 0}\right.$, meaning that initially only the sensitive subpopulation is dealt with, and that can be assumed in many cases) it will take the following form (Swierniak et al., 1998b):

$$
\begin{aligned}
& \dot{N}_{0}(t)= \\
& (1-2 u) \lambda N_{0}(t)-\alpha N_{0}(t)+d \alpha \int_{0}^{t} \phi_{1}(t-\tau) N_{0}(\tau) d \tau
\end{aligned}
$$

where

$$
\begin{aligned}
\phi_{1}(t) & =N_{1}^{1}(t) \\
& =\frac{1}{d} \sqrt{d / b} I_{1}(2 \sqrt{b d} t) \exp [-(b+d+\lambda) t] .
\end{aligned}
$$

A more general form of the integro-differential equation (14) with an arbitrary finite number of nonzero initial elements can be easily derived. Suppose that $N_{i}(0)=\delta_{i k}$ and $d \neq b$. Then

$$
\begin{aligned}
\dot{N}_{0}(t)= & (1-2 u) \lambda N_{0}(t)-\alpha N_{0}(t) \\
& +d \alpha \int_{0}^{t} \phi_{1}(t-\tau) N_{0}(\tau) d \tau+d \phi_{k}(t)
\end{aligned}
$$

and

$$
\begin{aligned}
\phi_{k}(t) & =N_{1}^{k}(t) \\
& =k \frac{1}{d}(\sqrt{d / b})^{k} \frac{I_{k}(2 \sqrt{b d} t)}{t} \exp [-(b+d-\lambda) t]
\end{aligned}
$$

Moreover, under the assumptions given above

$$
N_{\Sigma}(t)=N_{\Sigma}^{k}(t)+N^{+}(t)
$$

where $N_{\Sigma}^{k}(t)$ is given by $(10)$ and

$$
N^{+}(t)=\alpha \int_{0}^{t} N_{\Sigma}^{1}(t-\tau) N_{0}(\tau) d \tau
$$

In case the process starts with more than one type of resistant cells, the superposition principle can be applied and the final model takes the following form:

$$
\begin{aligned}
\dot{N}_{0}(t)= & (1-2 u) \lambda N_{0}(t)-\alpha N_{0}(t) \\
& +d \alpha \int_{0}^{t} \phi(t-\tau) N_{0}(\tau) d \tau \\
& +d \sum_{k \geq l} N_{k}(0) \phi_{k}(t)
\end{aligned}
$$

This can be applied to any case with a finite number of non-zero initial conditions which allows 

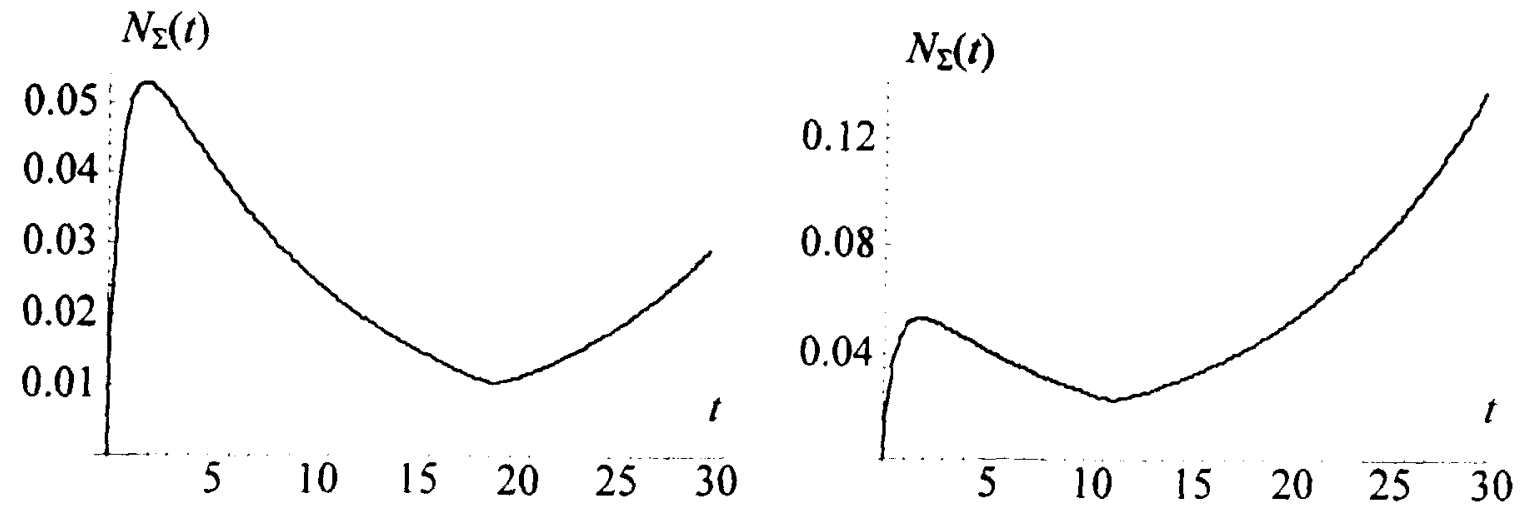

FIGURE 3 The dynamics of the resistant subpopulation for $\mathrm{M}=1, \mathrm{r}=0.1$ (left) and $\mathrm{r}=0.5$ (right)

the use of this form in any practical situation. The theoretical case of initial conditions with infinite support should be treated very carefully (see Świerniak et al., 1998c).

The constant treatment protocol given by (12) which guarantees decay of the cancer population after sufficiently long time is not realistic. Most of all, it does not take into account the cumulated negative effect of the drug upon normal tissues. To make the solution more realistic, the optimal control problem defined by the cost functional given in the form (13) may be solved. Further on, the necessary conditions for optimal control will be presented for model (16).

\section{NECESSARY CONDITIONS FOR OPTIMAL CONTROL OF THE POPULATION}

The optimization problem to be solved is to find a control $u(t)$ satisfying the constraint (6) for the system described by (20) minimizing the performance index (13).

A number of formulations of necessary conditions for the optimization problem for dynamical systems governed by integro-differential equations can be found in literature, e.g. (Bate. 1969: Connor, 1972; Gabasov and Kirilowa, 1971). However, they usually either are too general to be efficiently applied in such particular problem (bilinear model equation in which the control variable is beyond the integral, while the performance index is formulated in $L_{1}$ space) or have too strong constraints for example smoothness of the control function. Nevertheless, following the line of reasoning presented by Bate (1969), it is possible to find the solution to the problem.

It is important to notice that, although the performance index (13) seems to consist of two components a sum and an integral, the sum actually involves another integral which stems from (18). Therefore, it should be rewritten to emphasize this relation. Substituting (10) and (19) into (18) and, subsequently, into (13) we obtain:

$$
\begin{aligned}
J= & N_{0}(T)+N_{\Sigma}^{k}(T) \\
& +\int_{0}^{T}\left[\alpha N_{\Sigma}^{1}(T-\tau) N_{0}(\tau)+r u(\tau)\right] d \tau(21)
\end{aligned}
$$

where the function $N_{\Sigma}^{k}(t)$ is defined by $(10)$.

As mentioned before, applying Pontryagin maximum principle (Pontryagin et al., 1962) in a way similar to that shown in (Bate, 1969), the necessary conditions for optimal control are given by the folowing formulae:

$$
\begin{aligned}
u^{o p t}(t)= & \arg \min \left[p_{1}(t)\left(\alpha N_{\Sigma}^{1}(T-t) N_{0}(t)+r u(t)\right)\right. \\
& +2 p_{2}(t)\left((1-2 u(t)) \lambda N_{0}(t)-\alpha N_{0}(t)\right) \\
& \left.+d \alpha \int_{t}^{T} p_{2}(\tau) \phi_{1}(t-\tau) N_{0}(\tau) d \tau\right], \quad(22)
\end{aligned}
$$




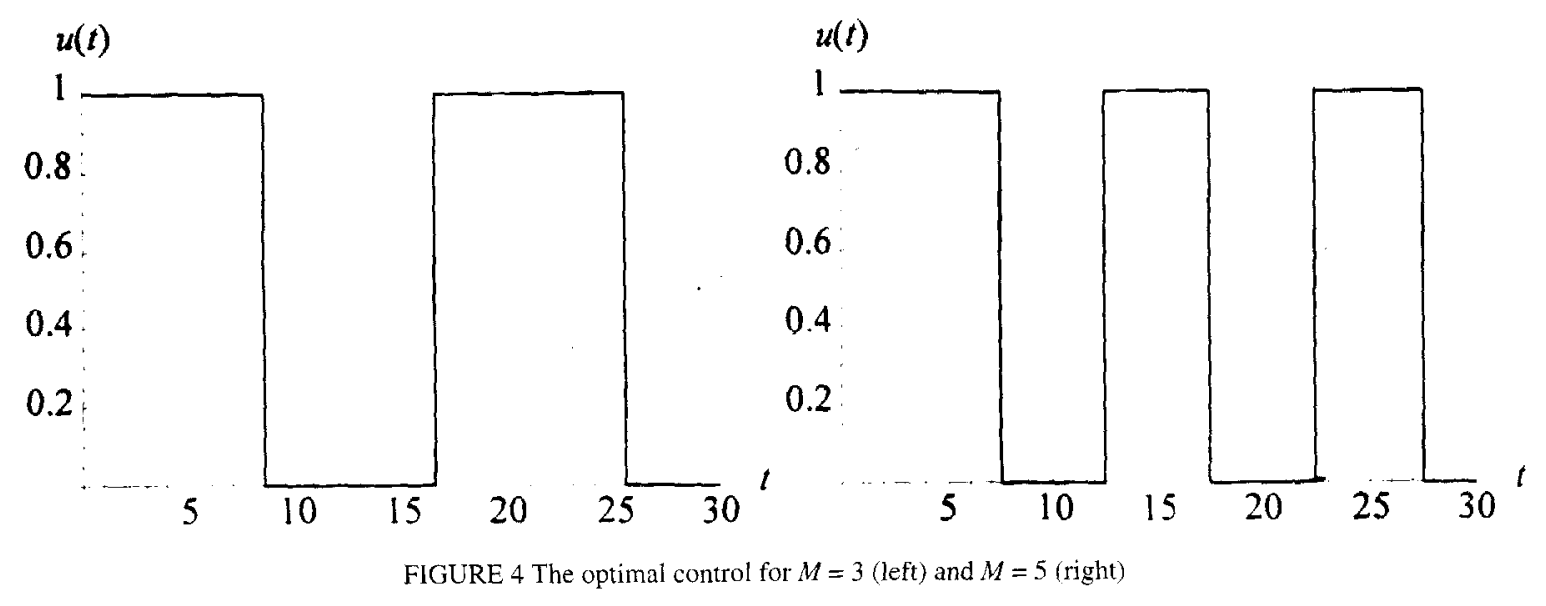

$$
\begin{gathered}
\dot{p}_{1}(t)=0 \\
\dot{p}_{2}(t)=-\alpha p_{1}(t) N_{\Sigma}^{1}(T-t) \\
+p_{2}(t)[(1-2 u(t)) \lambda-\alpha] \\
+d \alpha \int_{t}^{T} p_{2}(\tau) \phi_{1}(\tau-t) d \tau, \\
p_{1}(T)=1, \\
p_{2}(T)=1
\end{gathered}
$$

where (23), (24) are adjoint equations, $p_{1}(t), p_{2}(t)$ adjoint variables.

Moreover, denoting by

$$
\begin{aligned}
H= & p_{1}(t)\left(\alpha N_{\Sigma}^{1}(T-t) N_{0}(t)+r u(t)\right) \\
& +2 p_{2}(t)\left((1-2 u(t)) \lambda N_{0}(t)-\alpha N_{0}(t)\right) \\
& +d \alpha \int_{t}^{T} p_{2}(\tau) \phi_{1}(t-\tau) N_{0}(\tau) d \tau
\end{aligned}
$$

one may find without difficulty that:

$$
\Delta J=J(u+\delta u)-J(u) \approx \int_{0}^{T} \frac{\partial H}{\partial u} \delta u d t
$$

where $\Delta I$ and $\delta u$ are variational forms of $J$ and $u$, respectively. This will be a basis for development of a gradient method for finding solutions later on.

Since the adjoint equation (23) with given final condition (25) implies $p_{1}(t)=1=$ const and only a part of the right hand relationship depends on $u$ we are led to the condition in the form:

$$
u^{o p t}(t)=\arg \min \left[\left(r-2 p(t) \lambda N_{0}(t)\right) u(t)\right]
$$

where $p(t)=p_{2}(t)$ is a costate variable satisfying the following adjoint integro-differential equation:

$$
\begin{aligned}
\dot{p}(t)= & -\left[\alpha N_{\Sigma}^{1}(T-t)+p(t)[(1-2 u(t)) \lambda-\alpha]\right. \\
& \left.+d \alpha \int_{t}^{T^{\prime}} p(\tau) \phi_{1}(\tau-t) d \tau\right]
\end{aligned}
$$

with final condition:

$$
p(T)=1
$$

For further reference, let us denote

$$
H^{*}=\left(r-2 p(t) \lambda N_{0}(t)\right) u(t) .
$$

Furthermore, the condition (29) and the constraints (6) imply that, unless the problem is not singular, the optimal control should have a bang-bang form, i.e.:

$$
u^{o p t}(t)= \begin{cases}0 & \text { if } r-2 p(t) \lambda N_{0}(t)>0 \\ 1 & \text { if } r-2 p(t) \lambda N_{0}(t)<0\end{cases}
$$

which confirms suggestions of Harnevo and Agur (1992) who, having introduced a model which treats the emergence of drug resistance as a dynamic process, show how changes in the underlying assumptions affect the predictions about treatment efficacy. Their mathematical modeling results suggested that under gene amplification dynamics with high amplification probability, protocols involving frequent low-concen- 


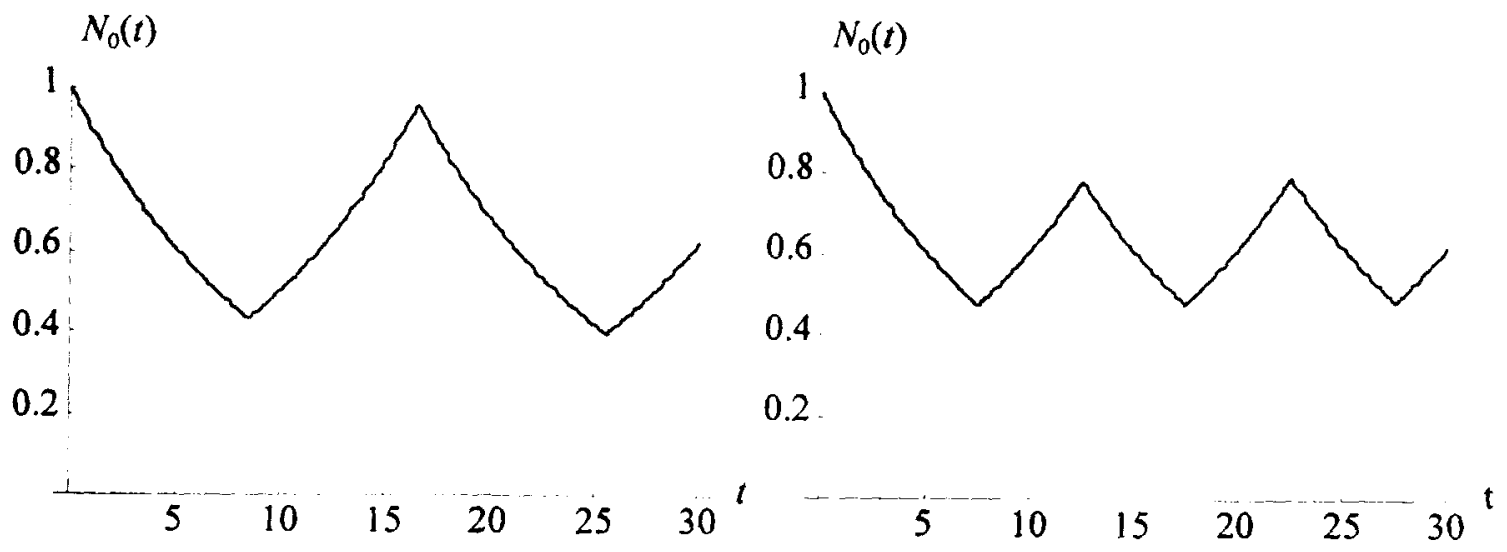

FIGURE 5 The dynamics of the sensitive subpopulation for $M=3$ (left) and $M=5$ (right)

tration dosing may result in the rapid evolution of large fully resistant residual tumors, whereas the same total doses divided into high-concentration doses applied at larger intervals may result in partial or complete remission (which was an alternative recommendation to that of Coldman and Goldie (1983)). The control law (33) implies the way a drug should be administered, with highest doses followed by "no-drug" periods. As a result, two questions arise: how many switches the control should have (i.e. how many times the highest dose should be given) and what are the optimal switching times for this number of switches (meaning how long any period of the chemotherapy protocol should last). An attempt to give an answer to both of those questions would be the most ambitious task, but so far no efficient method is known to the authors for dealing with such a complex problem. Even for finite-dimensional problems analytical solution is not available except for the second-order case when all solutions to the two-point boundary value problem arising from necessary conditions could be classified (Świerniak and Polański 1993; 1994). Moreover, even in this case nonexistence of singular solutions could not be guaranteed (Świerniak and Duda, 1994; Świerniak and Polański, 1994). Nevertheless, the approach proposed in this paper should give very satisfying results. In the subsequent section a numerical algorithm is proposeed that allows to find optimal switching times for a given number of switches, being assumed arbitrarily at the beginning of the algorithm. Afterwards, it is possible to use this method for any number of switches and compare obtained values of the performance index trying to find the best solution. Although this is not fully satisfactory in analytical terms, in case of infinite-dimensional systems, no firm conclusions can be drawn from the solution behaviour for different number of switches. However, it is always possible to define an upper limit for the value of number of switches, since control with too many switches is not applicable. Taking that into account, it should be sufficient to apply presented method in developing new treatment protocols.

\section{A GRADIENT METHOD FOR FINDING OPTIMAL CONTROL}

The algorithm presented here is a modified version of the ones proposed by Duda (1995; 1997), developed to solve an optimization problem in bilinear finite dimensional models.

Let us assume that the problem described by (20), (30), (31) and (33) is not singular and therefore the optimal control is a bang-bang process. Then, for an 


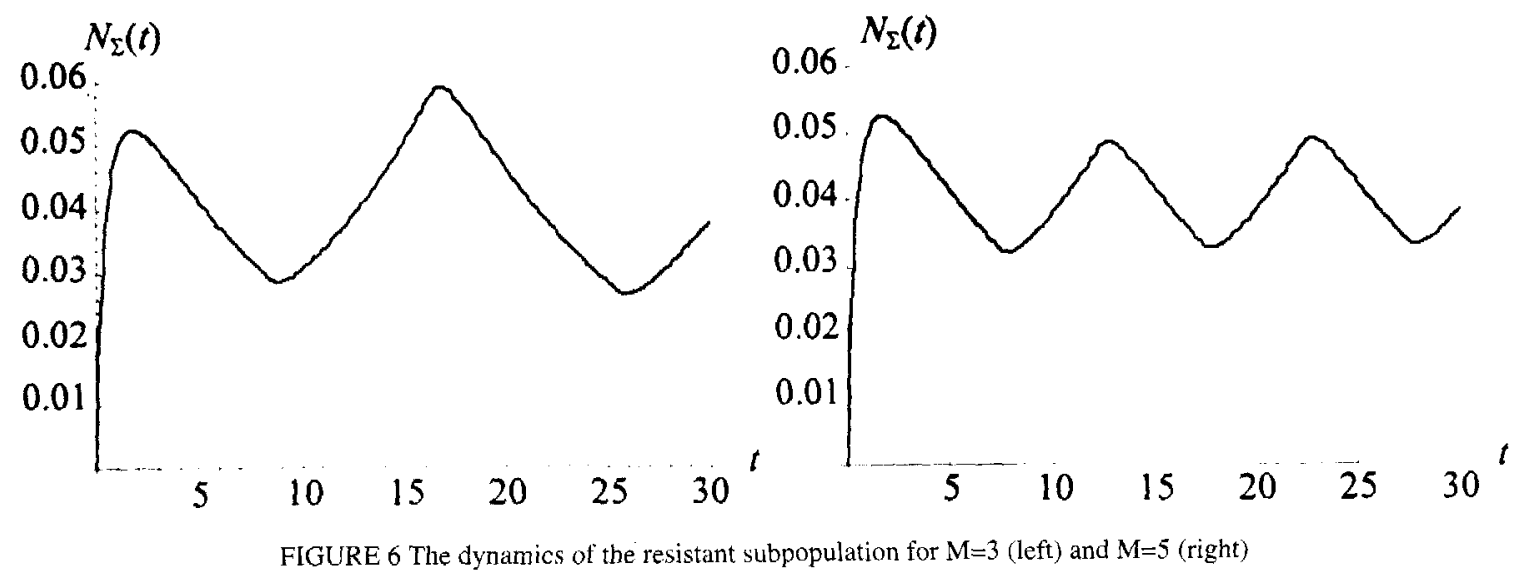

arbitrarily chosen odd number $M$ of switches the control variable $u(t)$ is given by:

$$
u(t)=\sum_{j=0}^{(M-1) / 2}\left[1\left(t-\tau_{2 j}^{M}\right)-1\left(t-\tau_{2 j+1}^{M}\right)\right]
$$

where $\tau_{j}^{M}$ are switching times $(j=1,2, \ldots, M), \quad \tau_{0}^{M}=0, \quad \tau_{M}^{M} \leq T$ and $\mathbf{l}(t)$ is the unit step function.

In a bang-bang process, a variation of control $\delta u$ at particular switching time $\tau_{j}^{M}$ is caused by a variation $\delta \tau_{j}^{M}$ :

$$
\delta u\left(\tau_{j}^{M}\right)=2 \delta^{*}\left(t-\tau_{j}^{M}\right) \delta \tau_{j}^{M},
$$

where $\delta *(\cdot)$ is the Dirac delta function. Hence, the variational form of the bang-bang process is as follows:

$$
\delta u(t)=2 \sum_{j=1}^{M}(-1)^{j+1} \delta^{*}\left(t-\tau_{j}^{M}\right) \delta \tau_{j}^{M}
$$

Substituting (35) into (28) yields:

$$
\Delta .\left.J \approx 2 \sum_{j=1}^{M}(-1)^{j+1} \frac{\partial H}{\partial u}\right|_{\tau=\tau_{j}^{M}} \delta \tau_{j}^{M}
$$

Since minimizing the performance index $J$ requires the variation $\delta \tau_{j}^{M}$ to satisfy $\Delta J \leq 0$, the most convenient way is to make $\delta \tau_{j}^{M}$ meet the following condition:

$$
\delta \tau_{j}^{M}=\left.(-1)^{j} k_{j} \frac{\partial H}{\partial u}\right|_{\tau=\tau_{j}^{M}}, \quad j=1,2, \ldots, M
$$

Therefore the numerical algorithm can be described in the following way:

1. Assume $M$ and initial values of $\tau_{j}^{M}, \quad j=1,2, \ldots, M$.

2. Solve the equation (16).

3. Solve the costate equation (30) with final condition (31)

4. Choose coefficients $k_{j}$ and calculate $\delta \tau_{j}^{M}$ using relation (37).

5. Calculate new switching times $\tau_{j}^{M}+\delta \tau_{j}^{M}$.

6. Repeat steps $2-5$ until the stop criterium is satis-

fied, i.e. $\sum_{j=1}^{M}\left(\delta \tau_{j}^{M}\right)^{2}<\varepsilon_{1}$ or $\Delta J<\varepsilon_{2}$, where $\varepsilon_{1}, \varepsilon_{2}$ are is a small given numbers.

Since the algorithm allows to find a solution for a given $M$ only, it seems reasonable to extend it and repeat all steps for different numbers of switches. This would enable to choose the best treatment in context of performance index (13) from protocols with different values of $M$ belonging to a bounded set. In the infinite dimensional system the solution behaviour with respect to $M$ for finite number of different values does not prove that there will not be better 
results for some greater $M$. Nevertheless, taking into account properties of a drug action and clinical reality one can assume an upper limit for its values.

Exemplary results obtained with this methods are shown on Figures 1-6, for parameters based on the research (Kaufman et al., 1981) $(b=0.2, d=2$, $\alpha=0.01, \lambda=0.1$ ). The control horizon (the length of the chemotherapy) has been set to $T=30$. Figures $1-3$ illustrate results for one switch, $M=1$, and two weighing factores $r$. The optimal control is presented on Figure 1, while Figures 2 and 3 show the growth of the sensitive and resistant subpopulations, respectively, for $r=0.1$ and $r=0.5$. The simulation starts with a sensitive population $\left(N_{i}(0)=0\right.$ for $\left.i \geq 0\right)$. Worth noticing is the fact that despite such initial conditions the resistant subpopulation appears. It is clear that choosing too big value of rleads to $u(t)=0$ whereas to small value of $r$ results in $u(t)=1$ for $0 \leq t \leq T$.

Figures 4-6 allow comparison of results for different number of switches $M=3$ and $M=5(r=0.1$ in both cases). Although they are slightly worse in terms of the value of the performance index $(J=2.38$ for both $M=3$ and $M=5$, comparing with $J=2.35$ for $M=1$ ), both subpopulations grow significantly less for greater number of switches, which might imply that a protocol involving greater number of switches might be more preferable, at least in some cases.

\section{CONCLUSIONS}

In this paper we discuss a method to develop treatment protocols in chemotherapy basing on results stemming from application of optimal control theory to the infinite dimensional model of evolution of drug resistance in cancer cell population.

Attempts at optimization of cancer chemotherapy using optimal control theory have a long history (see e.g. reviews in Swan, 1990; Swierniak, 1995). The idea has been criticized many times (see e.g. Shin and Pado, 1982; Tannock, 1978; Wheldon, 1988). Only simplest concepts have won attention in the medical world. These include the clonal resistance model (Goldie and Coldman, 1979) and the kinetic resistance theory by Norton and Simon (1977).
It has been shown that, most likely, the optimal protocol should have a bang-bang form, at least in some cases involving increasing drug resistance. This result is similar to those proposed in the works concerning drug resistance (Harnevo and Agur, 1992), or dealing with cell-cycle-phase dependent treatment (Duda, 1997; Swierniak, 1995). Its applicability in clinical trials is arguable, however it gives a solidly based qualitative picture of possibly optimal chemotherapy scheduling.

Yet another approach to the optimal control problem stated for the model (5) is to consider $N_{0}$ as a virtual control (which would be convenient if it was somehow possible to shape the sensitive subpopulation). Then the infinite-dimensional model (5) could be transformed into an integral form:

$$
\begin{aligned}
N_{\mathrm{I}}(t)= & k \frac{1}{d}(\sqrt{d / b})^{k} I_{k}(2 \sqrt{b d} t) e^{-(b+d-\lambda) t} \\
& +\int_{0}^{t} \phi_{1}(t-\tau) N_{0}(\tau) d \tau
\end{aligned}
$$

The optimal control problem for integral systems such as (38) is much simpler and could be solved using existing methods. However, the assumption about capability to shape $N_{0}$ arbitrarily is very strong and thus applicability of the method is arguable.

\section{Acknowledgements}

The research has been supported by the $\mathrm{KBN}$ grant 8T11E 03315.

\section{References}

Abramowitz, M. and I.A. Stegun (1964). Handbook of Mathematical Functions. National Bureau of Standards. Washington.

Axelrod, D.E., K.A. Baggerly and M. Kimmel (1993). Gene amplification by unequal chromatid exchange: Probabilistic modeling and analysis of drug resistance data. Joumal of Theoretical Biology 168, 151-159.

Bate, R.B. (1969). The optimal control of systems with transport lag. Advances in Control Systems 7, 165-224

Brown, P.C., S.M. Beverly and R.T. Schimke (1981). Relationship of amplified dihydrofolate reductase genes to double minute chromosomes in unstably resistant mouse fibroblasts cell lines. Mol. Cell. Biol. 1, 1077-1083.

Coldman, A.J. and J.H. Goldie (1983). A model for the resistance of tumor cells to cancer chemotherapeutic agents. Math. Biosci. $65,291$.

Connor, M.A. (1972). Optimal control of systems represented by differential-integral equations. IEEE Trans. on Automatic Control pp. 164-166. 
Duda, Z. (1994). Evaluation of some optimal chemotherapy protocols by using gradient method. Applied Mathematics and Computer Science, special issue: Control and Modelling of Cancer Cell Population 4, 257-263.

Duda, Z. (1995). A gradient method for application of chemotherapy protocols. Journal of Biological Systems 3: No. 1, 3-11.

Duda, Z. (1997). Optimal synchronization and recruitment protocols design via a gradient type method. In: Advances in Mathematical Population Dynamics - Molecules, Cells and Man. pp. 579-594. World Scientific.

Gabasov, R. and F.M. Kirilowa (1971). Qualitative Theory of Optimal Processes. Nauka. Moscow

Goldie, J.H. and A.J. Coldman (1979), A mathematical model for relating the drug sensitivity of tumors to their spontaneous mutation rate. Cancer Treat. Rep 63, 1727-1733.

Harnevo, L.E. and Z. Agur (1991). The dynamics of gene amplification described as a multitype compartmental model and as a branching process. Math. Biosci. 103, 115-138.

Harnevo, L.E. and Z. Agur (1992). Drug resistance as a dynamic process in a model for multistep gene amplification under various levels of selection stringency. Cancer Chemother. Pharmacol. 30, 469-476.

Hamevo, L. E and Z. Agur (1993). Use of mathematical models for understanding the dynamics of gene amplification. Mutat. Res. $292,17-24$

Kautman, R.J., PC. Brown and R. T. Schimke (1981). Loss and stabilization of amplifjed dihydrofolate reductase genes in mouse sarcoma s-180 cell lines. Mol. Cell. Biol. 1, 1084-1093.

Kimmel, M. and D.E. Axelrod (1990). Mathematical models of gene amplification with applications to cellular drug resistance and tumorigenicity. Genetics 125, 633-644.

Kimmel, M. and D.E. Axelrod (1994). Fluctuation test for two-stage mutations: Application to gene amplification. Mutat. Res. 306, 45-60.

Kimmel, M, and D.N. Stivers (1994). Time - continuous branching walk models of unstable gene amplification. Bull. Math. Biol. 56, 337-357.

Kimmel, M., D.E. Axelrod and G.M. Wahl (1992). A branching process model of gene amplification following chromosome breakage. Mutat. Res. 276, 225-240.

Morrow, J. (1970). Genetic analysis of azaguanine resistance in an established mouse cell line. Genetics 65, 279-287.

Murnane, J.P, and M.J, Yezzi (1988). Association of high rate of recombination with amplification of dominant selectable gene in human cells. Somatic Cell and Molecular Genetics 14 $273-286$.

Norton, L. and R. Simon (1977). Tumor size, sensitivity to therapy, and design of treatment schedules. Cancer Treat. Rep. 61 , 1307-1317.

Polański, A., M. Kimmel and A. Świerniak (1997). Qualitative analysis of the infinite-dimensional model of evolution of drug resistance. In: Advances in Mathematical Population Dynamics - Molecules, Cells and Man. pp. 595-612. World Scientific.
Pontryagin, L.S., V.G. Boltyanski, R.V. Gamkrelidze and E.F. Mischenko (1962). Mathematical Theory of Optimal Processes. Wiley. New York.

Shin, K,G. and R. Pado (1982), Design of optimal cancer chemotherapy using a continuous-time state model of cell kinetics. Math. Biosci. 59, 225-248.

Stark, G.R. (1993). Regulation and mechanisms of mammalian gene amplification. Adv. Cancer Res. 61, 87-113.

Swan, G.W. (1990). Role of optimal control theory in cancer chemotherapy. Math. Biosci. 101, 237-284.

Swierniak, A. (1995). Cell cycle as an object of control. Journal of Biological Systems 3:1, 41-54.

Świerniak, A., A. Polański, J. Śmieja and M. Kimmel (1996a). Modeling and control of drug resistance in chemotherapy. Proc. of 15 IASTED Conference Modeling, Identification. and Control pp. $1-4$

Świerniak. A.. A. Polański, J. Śmieja and M. Kimmel (1998a). Drug resistance as infinite dimensional control process with positive feedback. Proc. of 5 th Intern. Symp.: Methods and Models in Automation and Robotics 1, 285-289.

Świerniak, A., A. Polański, J. Śmieja and M. Kimmel (1998b) Modelling of drug resistance evolution - infinite dimensional ode versus integrodifferential approach. pp. 18-23. IASTED/ACTA Press. Honolulu.

Swierniak, A. and A. Polan'ski (1993). All solutions to the tpbvp arising in cancer chemotherapy. Proc. 7 Symp. System, Modeling, Control pp. 223-229.

Świerniak, A and A. Polański (1994). Irregularity of optimal control problem in scheduling of cancer chemotherapy. Appl. Math. and Comp. Sci., special issue: Control and Modelling of Cancer Cell Population 4, 263-271.

Swierniak, A. and Z. Duda (1994). Singularity of optimal control problems arising in cancer chemotherapy. Math. and Comp. Modeling 19. 255-262.

Świemiak, A., M. Kimmel and A. Polański (1996b). Control prob lems arising in chemotherapy under evolving drug resistance. Preprints 13 IFAC World Congress B, 411-417.

Swierniak, A., M. Kimmel and A. Polanski (1998c). Infinite dimensional model of evolution of drug resistance of cancer cells. Journal of Mathematical Systems, Estimation and Control 8: 1, $1-17$.

Tannock, I. (1978). Cell kinetics and chemotherapy: a critical review. Cancer Treat. Rep. 62, 1117-1133.

TIsty, T., B. H. Margolin and K. Lum (1989), Differences in the rates of gene amplification in nontumorigenic and tumorigenic cell lines as measured by luria-delbrück fluctuation analysis. Proc. Natl. Acad. Sci. USA 86, 9441-9445.

Varshaver, N.B., M.I. Marshak and N.I. Shapiro (1983). The mutational origin of serum independence in chinese hamster cells in vitro. International Journal of Cancer 31, 471-475.

Wheldon, T.E. (1988). Mathematical Models in Cancer Chemotherapy. Medical Sci. Series, Hilger. Bristol.

Windle, B. and G.M. Wahl (1992). Molecular dissection of mammalian gene amplification: New mechanistic insights revealed by analysis of very early events. Mutat. Res. 276, 199-224. 


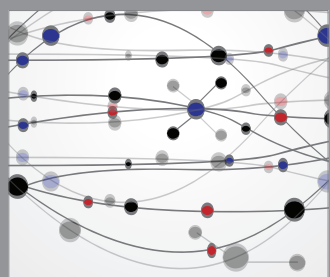

The Scientific World Journal
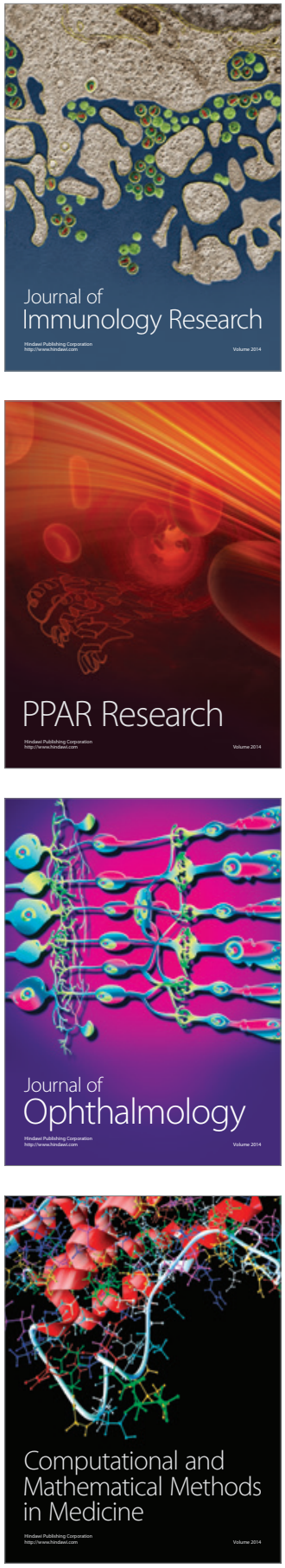

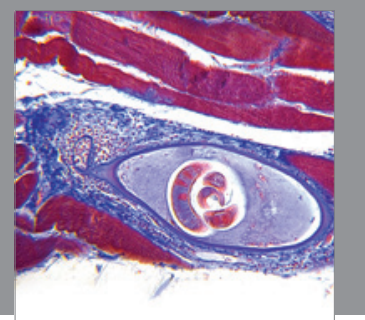

Gastroenterology

Research and Practice
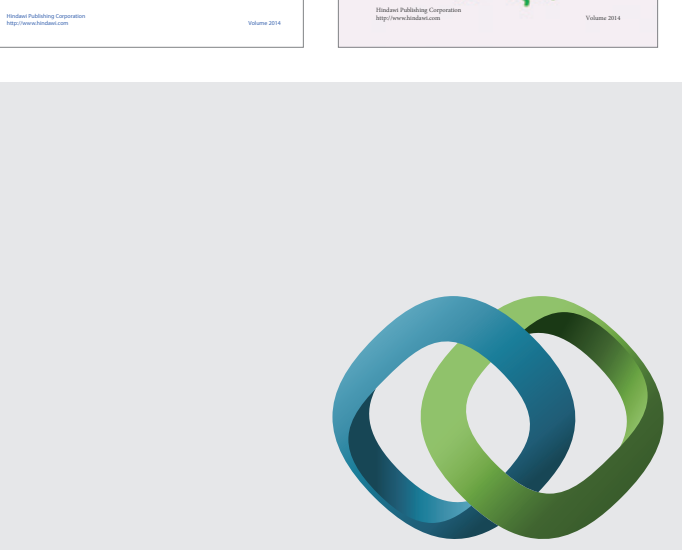

\section{Hindawi}

Submit your manuscripts at

http://www.hindawi.com
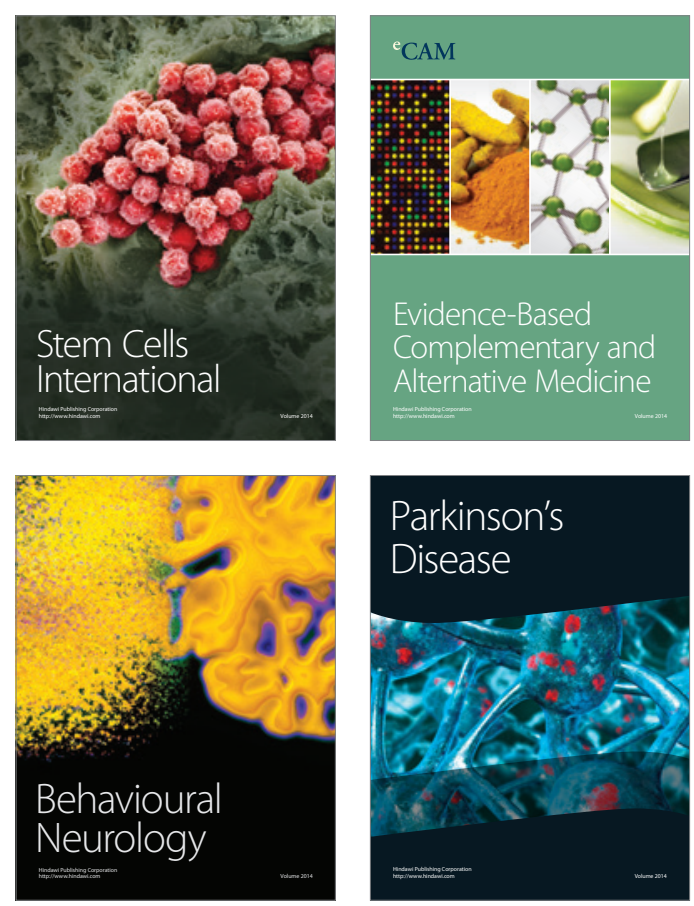

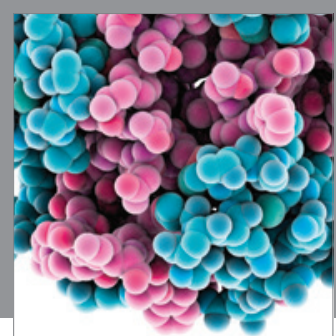

Journal of
Diabetes Research

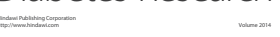

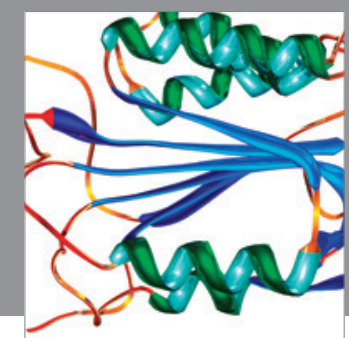

Disease Markers
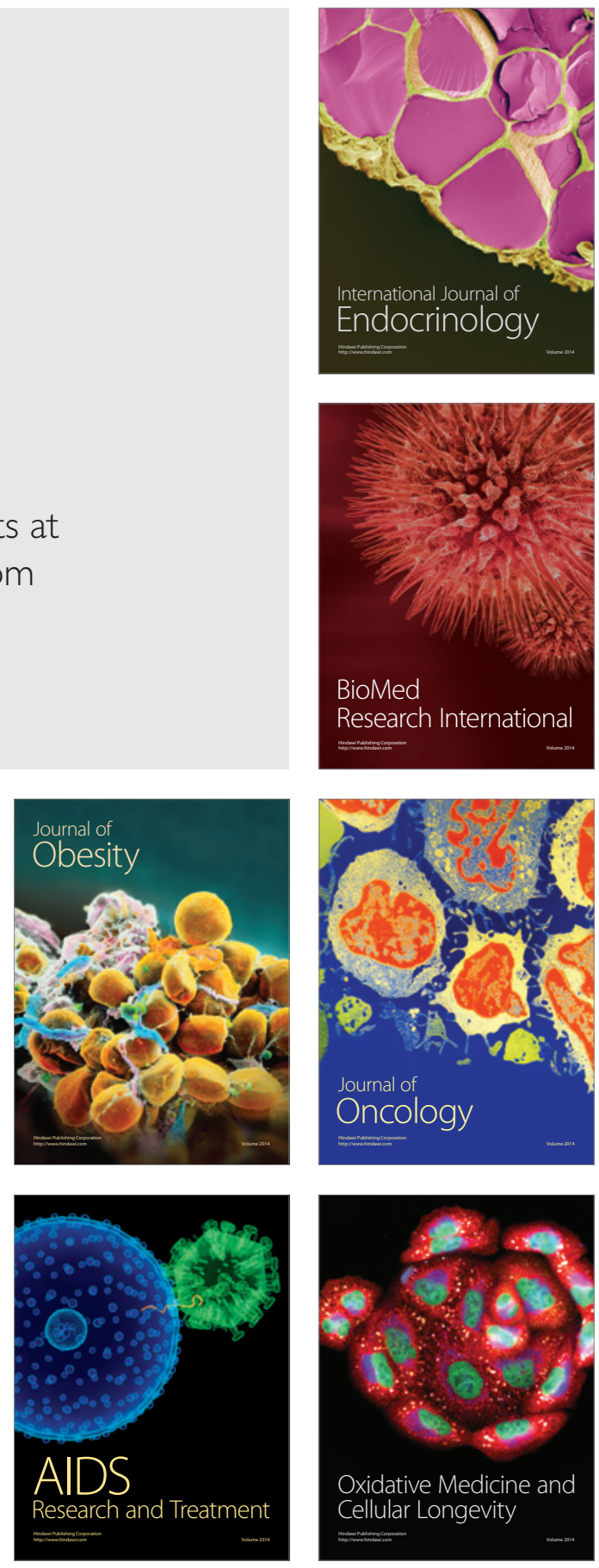